\title{
Recurrent dislocation of the hip in adult paraplegics
}

\author{
G P Graham FRCS, ${ }^{1}$ C M Dent FRCS,${ }^{2}$ P D Evans FRCS, ${ }^{1}$ B McKibbin FRCS $^{3}$ \\ ${ }^{1}$ Senior Registrar, ${ }^{2}$ Registrar, ${ }^{3}$ Professor, Department of Traumatic and Orthopaedic \\ Surgery, Cardiff Royal Infirmary, Newport Road, Cardiff CF2 1SZ, Wales.
}

\begin{abstract}
Recurrent dislocation of the hip is rare and has not previously been reported in adult paraplegics. This paper describes 3 cases. In one patient it was spontaneous, occurring 16 years after the original injury and was associated with a flexion-adduction contracture of the hip and a shallow acetabulum. One case occurred after minor trauma in a patient who experienced flexion-adduction spasms of the hip. In the third patient the condition was secondary to posterior acetabular deficiency following a conservatively treated fracture dislocation. All 3 patients experienced symptoms of disabling autonomic dysreflexia during the episodes of dislocation. The importance of recognising and adequately treating hip injuries in patients presenting with paraplegia secondary to spinal cord injury is stressed. In patients with spastic paraplegia presenting with recurrent dislocation of the hip, operative treatment combining a soft tissue repair and a bone block to augment the acetabulum is recommended.
\end{abstract}

Key words: spinal cord injury; paraplegia; hip; recurrent dislocation of hip.

\section{Introduction}

Although rare, recurrent dislocation of the hip in non paraplegics is now well described. ${ }^{1-5}$ It has been stated that paralysis is one of the predisposing factors in recurrent dislocation of the hip. ${ }^{3,5}$ However, while gluteal paralysis with preservation of flexor and adductor power is common in traumatic paraplegia, a review of the literature failed to discover previously reported cases of recurrent dislocation of the hip in this group of patients. McKibbin ${ }^{6}$ pointed out that spontaneous dislocation of the hip secondary to paralysis was almost always confined to children and that even severe muscle imbalance would not normally lead to instability in adults, unless the integrity of the acetabulum was compromised in some way, usually by injury or infection. The predisposition to spontaneous dislocation in children is attributed to the relative shallowness of the immature acetabulum. ${ }^{7}$ This paper reports 3 cases of

Correspondence: Department of Orthopaedic Surgery, Royal Childrens Hospital, Flemington Road, Parkville, Victoria 3052, Australia. recurrent dislocation of the hip occurring in adult paraplegics.

\section{Case reports}

Case 1

A 29 year old man who had been paraplegic for 6 months with a T5/6 fracture dislocation following a motorcycle accident fell out of his wheelchair on to his left hip. This caused no immediately apparent injury but over the succeeding 3 weeks he experienced episodes of autonomic dysreflexia causing sweating, tachycardia, and nausea, and during one of these attacks he noticed that his left hip looked deformed. Radiographic screening revealed posterior dislocation in adduction and internal rotation (Fig 1). The dislocation which occurred during screening caused an attack of autonomic dysreflexia. A CT scan of the hip showed that there was a small fragment of bone from the posterior part of the acetabulum. Because of the severe symptoms an operation to stabilise the hip was performed. At operation through a posterior approach the capsule of the hip joint was found to be stretched and redundant posteriorly and a false cavity had been formed. The redundant capsule was 


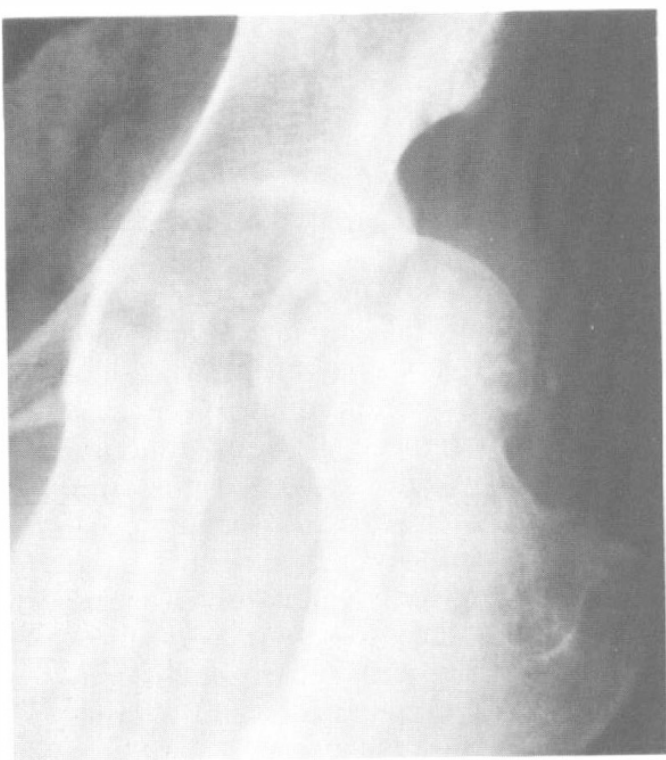

Figure 1 Radiograph taken during screening of the left hip in Case 1, showing posterior dislocation of the hip in adduction and internal rotation.

plicated and the false cavity was obliterated with 3 screws with washers. He was kept on bed rest for 5 weeks. During this time he experienced severe spasms which caused the hip to flex, adduct and internally rotate. Despite medication the spasms could not be controlled causing the repair to fail and the hip to sublux once more. A second operation was performed in which a complete obturator neurectomy and adductor and psoas tenotomies were combined with an open reduction through a posterior approach. A large piece of iliac crest was screwed on to the posterior acetabular wall to augment it (Fig 2). He was kept in abduction plasters for 6 weeks and flexion of the hip restricted for a further 2 weeks. The second procedure was successful and he has had no further problems with the hip one year after the operation.

\section{Case 2}

A 45 year old female who had been a $T 2 / 3$ paraplegic for 16 years gave a 9 month history of intermittent shortening of the left leg associated with a 'funny feeling' in the

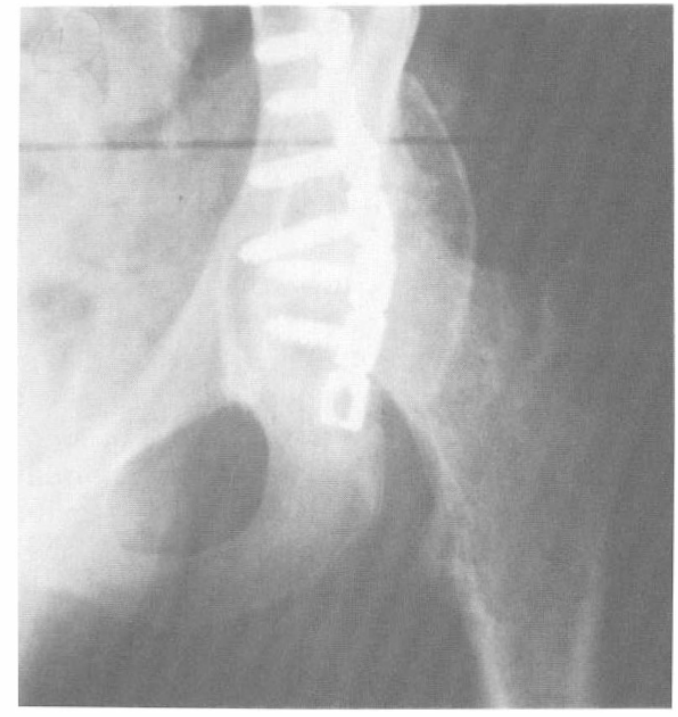

Figure 2 Postoperative radiograph of the left hip in Case 1 showing the acetabular augmentation.

left hip and episodes of sweating and nausea. This occurred especially when she transferred to her wheelchair and in bed at night. Examination revealed the left hip to have a mild flexion-adduction contracture and to be easily dislocatable in internal rotation. Radiographs confirmed that the left hip was dislocating and could be reduced with gentle traction (Fig 3). At operation the hip was found to be unstable. The capsule was stretched and voluminous and the femoral head dislocated easily into the cavity formed. There was no obvious bony deficiency although the posterior lip of the acetulum was eroded. In order to stabilise the hip an artificial Ligamentum Teres was made using a double strand of carbon fibre passing the carbon from the acetabulum through the femoral head. Postoperatively she was treated in an antirotation boot for 3 weeks, and was then allowed to mobilise unrestricted. No further episodes of dislocation have occurred 3 months after the procedure.

\section{Case 3}

A 26 year old female who had been a T4/5 paraplegic for 6 months complained of a 6 


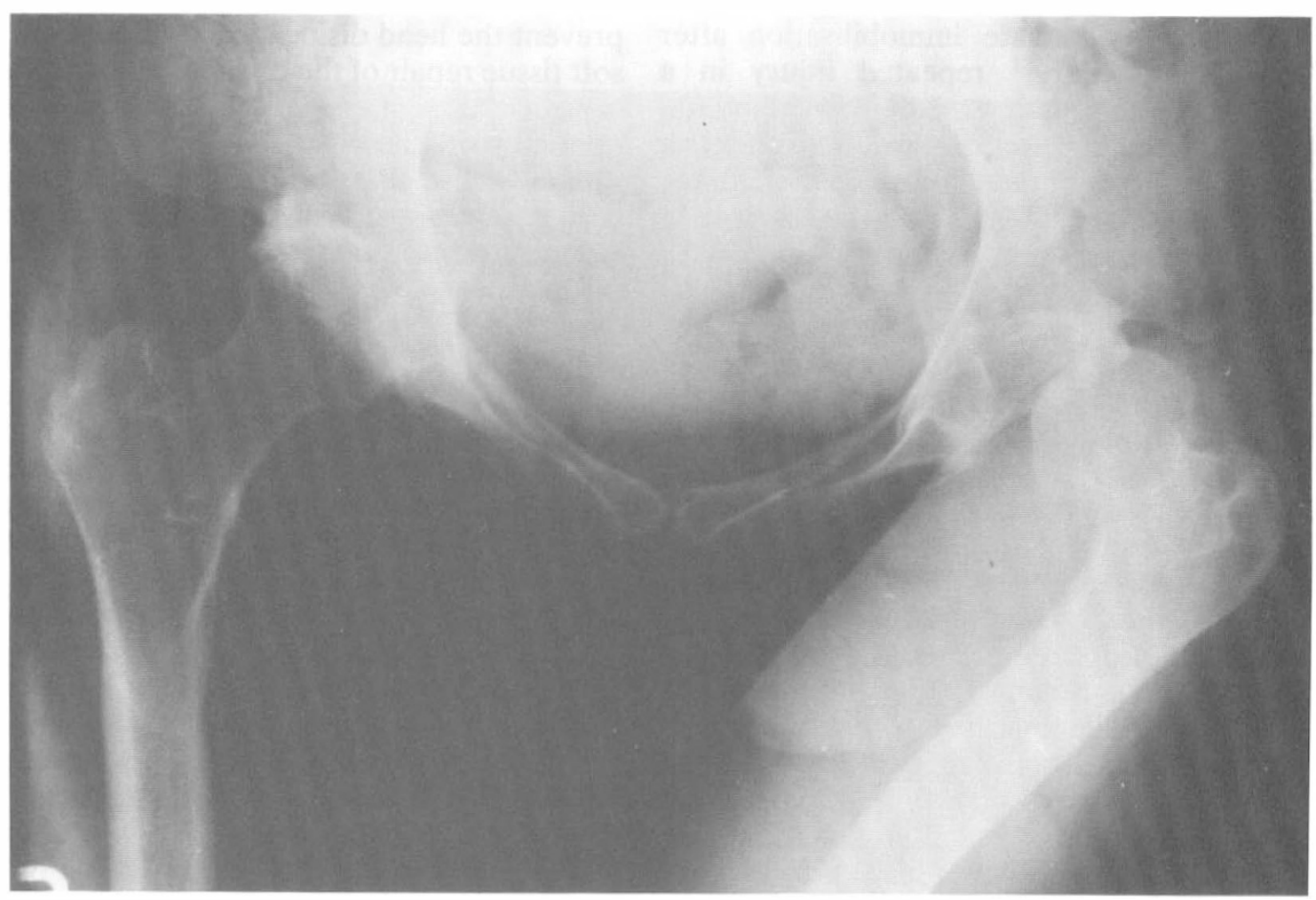

Figure 3 Radiograph of the left hip in Case 2 showing the adduction deformity and shallow acetabulum. The hip could easily be dislocated and reduced.

week history of recurrent episodes of autonomic dysreflexia causing nausea and sweating. During these episodes she also complained of 'discomfort' in her left hip. Her original injuries had included a posterior fracture-dislocation of the left hip which had been treated conservatively. On examination the hip subluxed in internal rotation and adduction. It was easily reduced with gentle traction. Radiographs revealed a large bone fragment from the posterior aspect of the acetabulum. At operation through a posterior approach a large fragment of bone was found to be detached from the posterior acetabular wall. This was mobilised and screwed back into place with 2 screws. The capsule of the hip joint was stretched and lax posteriorly and was plicated. Postoperatively she was nursed supine in an abduction pillow for 3 weeks and then allowed to mobilise in her wheelchair. She has had no further problems with the hip 4 years after the repair.

\section{Discussion}

Brav $^{1}$ found that in normal (ie non paraplegic patients) recurrent dislocation occurred in less than $1.5 \%$ of patients who suffered an acute dislocation of the hip. He reviewed 264 traumatic dislocations and noted only 4 cases of recurrent dislocation. In 3 of these cases the acetabular wall was defective due to an acetabular fracture. Only one case occurred without a fracture defect. Liebenberg and Dommisse 5 reported 2 cases and described a soft tissue abnormality analogous to that occurring in recurrent dislocation of the shoulder, with laxity of the capsule and a pocket into which the femoral head dislocated.

Various reasons for recurrent dislocation 
in non paraplegics have been put forward including inadequate immobilisation after the initial injury, ${ }^{1}$ repeated injury in a chronic alcoholic, ${ }^{5}$ a shallow acetabulum and deficient posterior rim $^{8}$ and delay in reduction of the initial dislocation. ${ }^{3}$

Of the 3 cases described here, patients 1 and 2 had sustained no bony hip injury in the original accident. This was confirmed by review of their original radiographs. Patient 1 had experienced minor trauma to the hip when he fell out of his wheelchair 6 months after the original accident and this appeared to trigger the episodes of dislocation. He was prone to flexion-adduction spasms of his hips which were difficult to control and it is likely that the spasms, associated with the minor injury, precipitated the episodes of recurrent dislocation. Patient 2 was 16 years post injury and had experienced no trauma to the hip. The episodes of recurrent dislocation occurred spontaneously and the reasons for dislocation at this late stage are unclear. She did have a mild flexion-adduction deformity of the hip and adaptive changes in the acetabulum which was shallow (Fig 3). It is likely that a combination of these 2 factors caused the instability. In patient 3 the original injury had included a posterior fracture dislocation of the hip with a large posterior fragment. This had been treated conservatively on traction for 4 weeks. Reduction and internal fixation of the fracture had not been performed because of the paraplegia. A large defect in the posterior acetabular wall had been left by the imperfect reduction allowing the femoral head to dislocate.

During the episodes of dislocation all 3 of our patients experienced unpleasant symptoms of nausea, tachycardia, sweating and hypertension. This was due to autonomic dysreflexia, a syndrome which can occur in patients with high thoracic or with a cervical cord injury. ${ }^{9}$ It is normally triggered by noxious stimuli. These unpleasant symptoms were quite disabling and worrying for the patients. Transferring to and from their wheelchairs was difficult as this tended to cause dislocation and brought on the dysreflexic symptoms.

Two main operative methods of repair have been described in non paraplegics.
Most authors use either a bone block to prevent the head dislocating ${ }^{5,8}$ or perform a soft tissue repair of the capsule, plicating the capsule to tighten it. $^{3}$ The findings at operation in the first 2 patients were the same as those described by other authors in non paraplegic patients. The capsule was stretched and attenuated and a recess had formed into which the femoral head could dislocate. There was no major bony deficiency. In patient 1 , in whom there was only a very small piece of bone avulsed from the posterior acetabular wall, a capsular plication was performed. The muscular spasms which he experienced postoperatively caused the hip to adduct, flex and internally rotate putting pressure on the repair and causing it to fail. The second procedure which involved an obturator neurectomy, psoas and adductor tenotomy and augmentation of the posterior acetabular wall with a bone block was successsful in preventing further dislocation (Fig 2). In patient 2 capsular plication and reconstruction of the ligamentum teres with carbon fibre was performed. The short term result of this procedure has been good and hip stability has been restored without complications. The recurrent dislocation was solved in patient 3 by restoring the bony anatomy.

\section{Conclusions}

In patients with traumatic paraplegia if symptoms of autonomic dysreflexia occur and the cause of the symptoms is not obvious the hips should be examined and if necessary radiographs taken to exclude dislocation.

As is illustrated in patient 3 , it is important that hip injuries in association with spinal injuries are recognised at the initial presentation. Fracture dislocation of the hip in paraplegic patients should be treated in the same way as in neurologically intact patients, and if necessary by open reduction and internal fixation.

In patients with spastic paraplegia with recurrent dislocation of the hip it is unlikely that a soft tissue 'double breasting' repair of the capsule alone, as recommended in non paraplegics, will work. It should be combined with a bone block to augment the 
acetabulum. If flexion-adduction spasms are a feature preoperatively an adductor teno- tomy and anterior obturator neurectomy should also be performed.

\section{References}

1 Brav EA (1962) Traumatic dislocation of the hip. J Bone Joint Surg 44A: 1115.

2 Dall D, McNab I, Gross A (1970) Recurrent anterior dislocation of the hip. J Bone Joint Surg 52A: 574-576.

3 Liebenberg F, Dommisse GF (1969) Recurrent post-traumatic dislocation of the hip. J Bone Joint Surg 51B: 632-637.

4 Lutter LD (1973) Post-traumatic hip redislocation. J Bone Joint Surg 55A: 391-394.

5 Sullivan CR, Bickel WH, Lipscomb PR (1955) Recurrent dislocation of the hip. J Bone Joint Surg 37A: 1266.

6 McKibbin B (1973) The use of splintage in the management of paralytic dislocation of the hip in spina bifida cystica. J Bone Joint Surg 55B: 163-172.

7 Ralis Z, McKibbin B (1973) Changes in shape of the human hip joint during its development. Their relation to its stability. J Bone Surg 55B: 780-785.

8 Townsend RG, Edwards GE, Bazant FJ (1969) In: Proceedings of the Canadian Orthopaedic Association. J Bone Joint Surg 51B: 194.

9 Lindan R, Joiner E, Freehafer AA, Hazel C (1980) Incidence and clinical features of autonomic dysreflexia in patients with spinal cord injury. Paraplegia 18: 285-292. 